\title{
Research on Systematic Design and Installation Based on Explosion Protection Technology Combined with Traction Elevator
}

\author{
Changming $\mathrm{Li}^{1}$, Qibing Wang ${ }^{1, \mathrm{a}}$, Yonggang Leng ${ }^{1}$ and $\mathrm{Yu} \mathrm{Li}^{2}$ \\ ${ }^{1}$ Tianjin University, Tianjin, China, 300072; \\ 2 Zhejiang Machinery Industry Information Research Institute, Zhejiang, China, 310009
}

\begin{abstract}
According to GB, NEC, ASME17.1, ANSI A10.4 and EN 81.1 standard, there is a danger of explosive gas or dust. And then for elevator explosion protection, we design a device type up to requirements of NEC Class 1, Division 1, Group C\&D as well as NEC Class 1, Division 2. It is also a controllable device type working well in highly explosive and hazardous work areas. We keep the GEDA explosion-proof lift fully fixed to protect workers and materials to the maximum extent. In addition, explosion-proof elevators in China must meet the standards of GB3836.1/2, GB50257, GB7588, etc. Combining the engineering practice over the years, the authors put forward in this paper a method of design and development as well as components based on explosion protection combined with traction elevator, which opens a new window for the systematic design and installation optimization of explosion-proof elevator.
\end{abstract}

\section{Preface}

Flammable and explosive dangerous media is common in the production environment of industries such as coal, oil, metallurgy, chemical, textile and pharmaceutical. With rapid development of these industries and people's increasing safety awareness of explosion protection in China in recent years, electrical apparatus for explosive atmospheres is widely used in relevant hazardous areas. For example, explosion-proof traction passenger-freight elevator and explosion-proof hydraulic passenger-freight elevator have become standard equipment in all explosion-proof places of industrial categories such as cereal, food, pesticide, fertilizer, wood processing, mine, textile, papermaking rubber, plastics, chemical, etc. [1] However, there are not many enterprises focusing on the research, development and production of explosion-proof elevator in China, which are also uneven. What's worse, explosion-proof elevators are different from ordinary passenger-freight elevators in performance and requirements, so the operation place and some standardized technical requirements for ordinary passenger-freight elevators can not be fully applied to explosion-proof elevators. Combining the engineering practice over the years, the authors briefly analyze and discuss the particularity of explosion-proof elevator in this paper in the case of explosion-proof traction elevator design, which applies to explosive gas environment area 1 and IIBT4.

\section{Design and Installation of Electrical Components}

${ }^{\mathrm{a} C}$ Corresponding author : 43410697@qq.com 


\subsection{Design of Electrical Components}

\subsubsection{Design of Electric Control Cabinet}

As the core of explosion-proof elevator design, electric control cabinet, located in the elevator room, decides whether the manufacturer has the ability to design and produce explosion-proof elevators. First of all, it is required to design the shell of the electric control cabinet to meet requirements of dIIBT4. Its strength and explosion-proof surface design must meet the requirements of GB3836.1/2. The larger the net volume of the shell, the longer the flameproof joint surface L, which means the strength requirements of the shell are higher. As there are a lot of parts in the electric control cabinet for elevators, if we design it into a single-cavity structure, then taking into account the requirements of the strength of the shell and the length of the flameproof surface, the control cabinet shell is bound to be very thick and then its weight as well as cost will be quite large. [2] Therefore, we can design it into a two-cavity structure, one for wiring and the other one for placing the intrinsically safe circuit, control circuit, inverter and so on. As the inverter is installed in the aluminum alloy control cabinet, the temperature rise will be relatively high. As for this, we can install an aluminum type radiator in the upper part of the control cabinet so that we can ensure that the inverter works normally in the explosion-proof control cabinet and that the elevator will not be affected by the poor heat dissipation. It is notable that the explosion-proof elevator control circuit has the requirements of the intrinsically safe circuit, which not only needs to meet requirements of the intrinsically safe circuit in the explosion-proof terminology, but also must meet the requirements of the elevator function essential safety.

\subsubsection{Design and Selection of Other Electrical Components}

For most parts of the explosion-proof elevator, we can use the existing mature explosion-proof products, for example, traction machine with explosion-proof three-phase asynchronous motor, explosion-proof telephone, explosion-proof brakes, door machines, fans and so on. However, we have to take the three points into consideration during our design:

First of all, the type of explosion protection shall apply to hazardous areas in Zone 1, so we can select the type according to the regional category based on Article 5.2 in GB3836.15-2000 standard.

Secondly, select the type according to temperature group [3].

Table 1. Relationship among Temperature Group, Surface Temperature and Ignition Temperature

\begin{tabular}{|c|c|c|}
\hline $\begin{array}{c}\text { Temperature group of the } \\
\text { electrical equipment }\end{array}$ & $\begin{array}{l}\text { Maximum surface temperature of the } \\
\text { electrical equipment }\end{array}$ & $\begin{array}{l}\text { Ignition temperature of } \\
\text { gas or steam }\end{array}$ \\
\hline T1 & $450^{\circ} \mathrm{C}$ & $>450^{\circ} \mathrm{C}$ \\
\hline $\mathrm{T} 2$ & $300^{\circ} \mathrm{C}$ & $>300^{\circ} \mathrm{C}$ \\
\hline T3 & $200^{\circ} \mathrm{C}$ & $>200^{\circ} \mathrm{C}$ \\
\hline T4 & $135^{\circ} \mathrm{C}$ & $>135^{\circ} \mathrm{C}$ \\
\hline T5 & $100^{\circ} \mathrm{C}$ & $>100^{\circ} \mathrm{C}$ \\
\hline T6 & $85^{\circ} \mathrm{C}$ & $>85^{\circ} \mathrm{C}$ \\
\hline
\end{tabular}

As shown in the table 1, equipment T4-T6 applies to dangerous places T4 requires.

Thirdly, select the type according to equipment category.

Table 2. Relationship between Gas/Steam Classification and Equipment Category 


\begin{tabular}{|c|c|}
\hline Gas/steam classification & Equipment category \\
\hline IIA & IIA, IIB or IIC \\
\hline IIB & IIB or IIC \\
\hline IIC & IIC \\
\hline
\end{tabular}

As shown in the table 2, equipment graded IIB and IIC applies to dangerous places IIB requires.

There are also some simple electrical parts or components, such as trip switches, limit switches and so on. For these components, it's not necessary to use mature explosion-proof products because they are just a switch contact, with only resistance circuit internally. According to requirements of GB3836.4-2010 "explosive environment Part 4: equipment protected by the intrinsically safe 'I'", power supply directly though explosion-proof safe shed is enough to meet the requirements of explosion protection. [4] As this type of components is in large consumption in explosion-proof elevators, this design will thus save a lot of cost.

\subsection{Installation of Electrical Components}

The installation of explosion-proof electrical equipment shall comply with the requirements of GB50257-1996 "Codes for construction and acceptance of electrical devices in electrical device installation project explosion and fire hazardous environment" and GB3836.15-2000 "Electrical equipment in explosive gas atmospheres (Part 15 - electrical installation in dangerous places (except coal mines) ". According to the authors' years of experience, the following questions are common in the installation of explosion-proof electrical equipment:

- The intrinsically safe wiring doesn't use shielded cable and is not marked blue.

- Flameproof, increased safety products tightening nuts are mixed up and redundant port is not effective blocked.

- Use raw tape on the explosion-proof surface to meet the sealing requirements.

- The safe shed is placed in the safety-type junction box.

- The metal housing reserved for the ground is not earthed.

The basic electrical equipment made by Ex i explosion-proof technology has the advantages of light weight, small size, low cost, electrical maintenance and application in hazardous area 0 , so this technology is the best protection technology in fieldbus. It is the trend of the fieldbus practice theory and overcomes the restriction of the Ex i explosion-proof fieldbus. [5]

\section{Explosion-Proof Design of Non-Electrical Parts}

\subsection{Requirements for Materials}

The selection of materials should be taken to prevent the occurrence of mechanical sparks and static electricity, which requires the supplier to provide the necessary material reports or supporting documents, such as the selection of materials for the floor of the room, the pit, the car and the car wall. [6]

\subsection{Design of Speed Governor}

Explosion-proof elevators are more and more widely used in the flammable and explosive places. Due to the particularity of the environment, in the design of explosion-proof elevators, various methods need to be taken to achieve the purpose of explosion protection, such as flameproof and intrinsically safe circuit. In addition, it is important to pay special attention to the collision and friction between the various parts of the elevator. Apart from the elevator traction pulleys and lifting ropes, the speed governor pulleys and ropes are also the main components that are prone to generate heat when friction 
occurs. The governor-tensioning device shall contain a device for preventing the wire rope from being disengaged and the device shall be of a sparkless material. The tensioning device shall be set to ensure that the tensioning device will not strike the ground when the rope is broken. [7] And the actual temperature can be achieved through the fast inverse algorithm as a boundary condition. The proposed method applies to a composite temperature sensor with inner and outer two-layer sheath and composite heat transfer process. [8]

\subsection{Design of Buffer}

A spring bumper or polyurethane bumper can be used and the rubber cushion can be added to the surface of the spring bumper against the counterweight or car. [9]

\subsection{Design of Other Parts}

Measures to prevent sparks shall be taken for parts with a potential risk of spark in normal use, such as traction wheel, guide wheel and reverse sheave rope, brake block and clamp, safety tongs, shaft of shift mechanism, trigger plate used when the safety gear hits the safety contact, boots lining of the sliding boots, one of the two parts of the impacting door locks, door hanging wheel, door block wheel, door guide shoes, etc. [10-13]

\section{Conclusions}

Explosion-proof elevator is a kind of special lifting equipment used in special environment. This article briefly describes the necessity of explosion-proof elevators in the design and supporting of explosion protection. Some of the design and supporting requirements described in this article are optional and can provide a useful reference for the design and use of explosion-proof elevators, which will therefore help to enhance China's development, production and use of explosion-proof elevator and promote the healthy and rapid development of explosion-proof elevator industry.

\section{References}

1. Heyan Wang, Liyong Ma, Biao Li and Sun Tao. A Study on Running-in Quality Evaluation Method of Power-shift Steering Transmission Based on Performance Parameter Testing [J] Lubrication Engineering 3 (2008): 025.

2. Jianguo Kong, Weirong Zhu, Wubiao Jiang. Design of the Dual-PWM Control Energy Feed-back Elevator Drive System Based on DSP and IPM [J] Electric Drive 8 (2007): 004.

3. General Administration of Quality Supervision, Inspection and Quarantine of the People's Republic of China (AQSIQ), China National Standardization Management Committee, GB3836.15-2000 Electrical equipment in explosive gas atmospheres (Part 15 - electrical installation in dangerous places (except coal mines)

4. General Administration of Quality Supervision, Inspection and Quarantine of the People's Republic of China (AQSIQ), China National Standardization Management Committee, GB3836.4-2010 explosive environment Part 4: equipment protected by the intrinsically safe ' $I$ '

5. Hua F. U. and L. I. U. Yin-xia. The Ex i Explosion-Proof Technology in the Fieldbus System [J]. Electric Explosion Protection 3 (2003): 005.

6. Hongwu Wang, Shaohua Liu. Application of PLC and Inverfer on Elevator [J] Electrical Engineering 1 (2007): 013.

7. Q. P. Zhang and J. R. Wan, Simulation of Generated Heat by Friction of Explosion-Proof Elevator Ropes, Advanced Materials Research, Vol. 853, pp. 413-420, 2014 
8. Hua Zhang, Zhao Wenzhu, and Wang Guodong. Research on dynamic temperature measurement method based on transient forward and inverse heat transfer model. Chinese Journal of Scientific Instrument 11 (2011): 016.

9. General Administration of Quality Supervision, Inspection and Quarantine of the People's Republic of China (AQSIQ), TSG T7011-05, Test rules of elevator buffer types

10. General Administration of Quality Supervision, Inspection and Quarantine of the People's Republic of China (AQSIQ), TSG T7012-05, Test rules of elevator door lock types

11. General Administration of Quality Supervision, Inspection and Quarantine of the People's Republic of China (AQSIQ), TSG T7010-2004, Test rules of elevator safety gear types (draft)

12. General Administration of Quality Supervision, Inspection and Quarantine of the People's Republic of China (AQSIQ), TSG T7009-05, Test rules of elevator speed governor types

13. General Administration of Quality Supervision, Inspection and Quarantine of the People's Republic of China (AQSIQ), TSG T7003-2011, Rules of supervised inspection and periodic inspection of elevators - explosion-proof elevator 